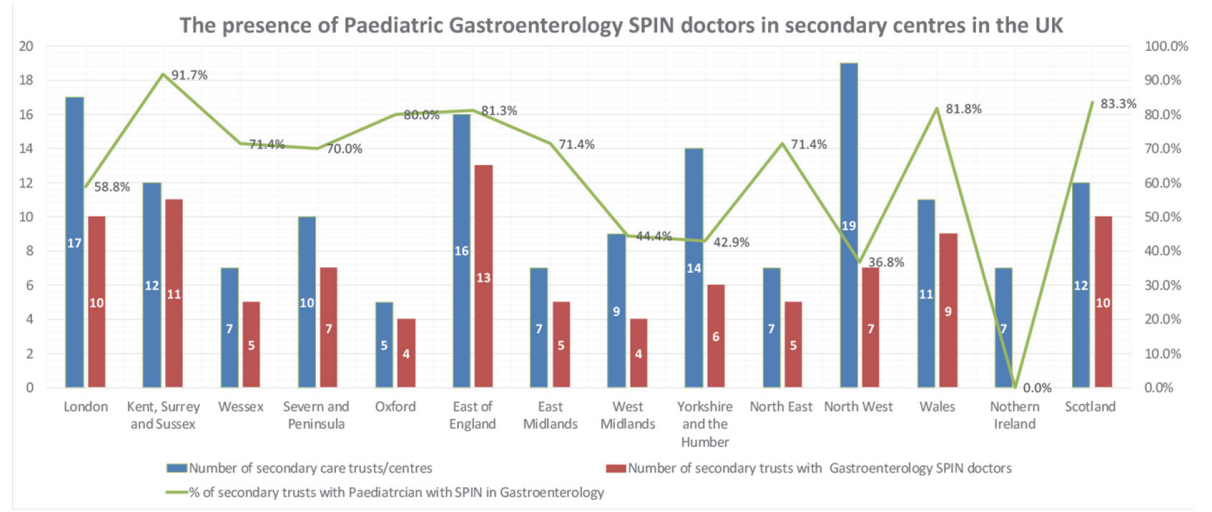

Abstract 010 Figure 1

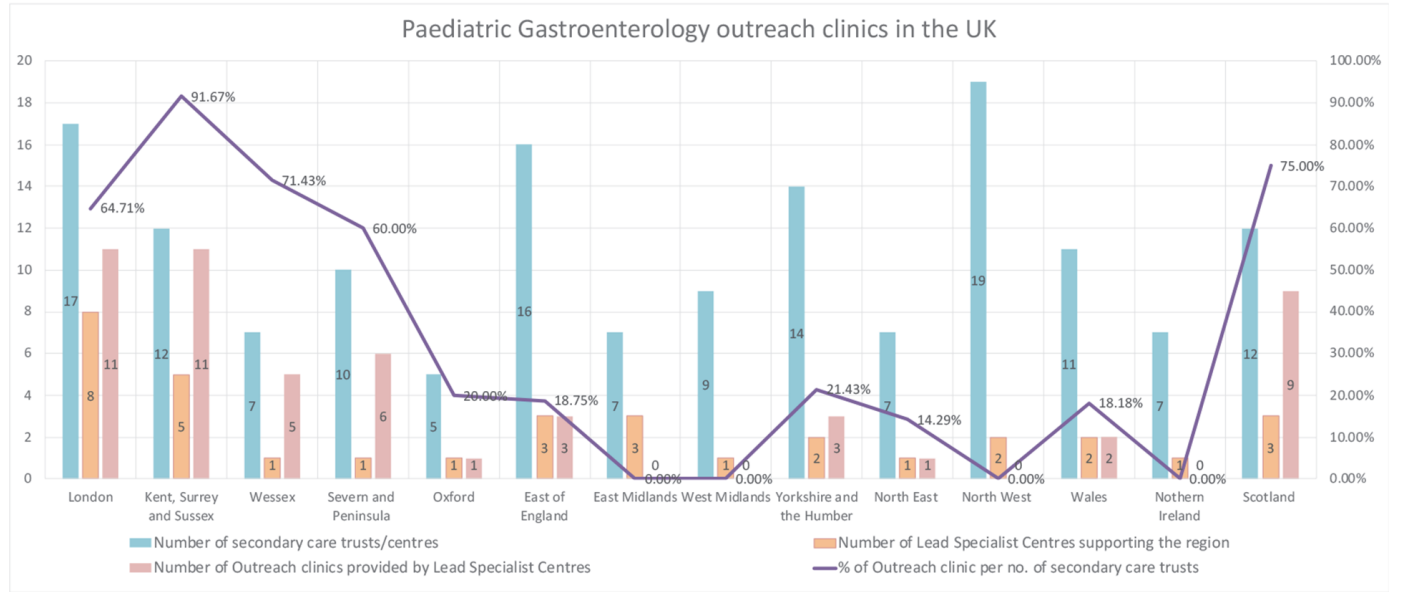

Abstract 010 Figure 2

across the UK. Approximately $62.7 \%$ of these centres had at least one Consultant Paediatrician with SPIN in Gastroenterology. The region with the largest percentage of secondary centres with SPIN doctors was KSS (Kent, Surrey, Sussex) with $91.7 \%$, followed by Scotland with $83.3 \%$. On the other hand, Northern Ireland and the North West of England had the lowest percentages. Southampton, Chelsea and Westminster and Bristol are the LSC which provide the highest number of outreach support. However, nearly a $1 / 3$ of the LSC in the UK do not provide any outreach clinics.

Discussion/Conclusion The results of this pioneering project highlight the wide variance in availability of SPIN doctors and outreach clinics in different regions across the UK. The lack of significant correlation amongst different analysed variables may suggest that this variability is secondary to unquantifiable factors such as geographical reach/constraints, intent and local funding policies. We believe this information is valuable to local, regional and national service commissioning groups in the redirection of efforts and resources to target populations where more urgent intervention is required. Points can be learnt from the top performing regions to improve delivery and establish uniformity of care. This project not only identifies the need for continued work in this domain, but also provides a foundation and structure for further analysis of the current services offered in secondary care.

\section{ACHALASIA CARDIA MANAGEMENT- CHANGING EXPERIENCE OF A TERTIARY PAEDIATRIC GASTROENTEROLOGY CENTRE}

Kwang Yang Lee, Dharamveer Basude. Bristol Royal Hospital for Children

\subsection{6/flgastro-2021-bspghan.11}

Background Achalasia is a primary oesophageal motor disorder of unknown aetiology. Incidence of 0.18/100,000 extrapolates to 23 new presentations per year in the UK. An ESPGHAN Survey revealed that $76 \%$ present to gastroenterology services. Management expertise is shared between paediatric gastroenterologists and paediatric surgeons.

Recent evidence shows that serial pneumatic balloon dilatations (PD) is equally effective compared to Laparoscopic Heller's myotomy with Dor fundoplication (HM) for type 1 and type 2 Achalasia. Peroral Oesophageal Myotomy (POEM) is most effective in Type 3 and resistant Achalasia and is not widely available. This is reflected in American College of Gastroenterology and European Society of Gastrointestinal Endoscopy guidelines. However, few UK paediatric gastroenterology, hepatology and nutrition (PGHAN) centres offer pneumatic balloon dilatations and hence patients may have limited options.

The Bristol PGHAN service has offered Pneumatic balloon dilatation as an option from 2010. Over time, practice has evolved from ad hoc dilatations to serial dilatations with a management algorithm. Families frequently choose PD as it is 
Current Algorithm for the Management of Paediatric Achalasia

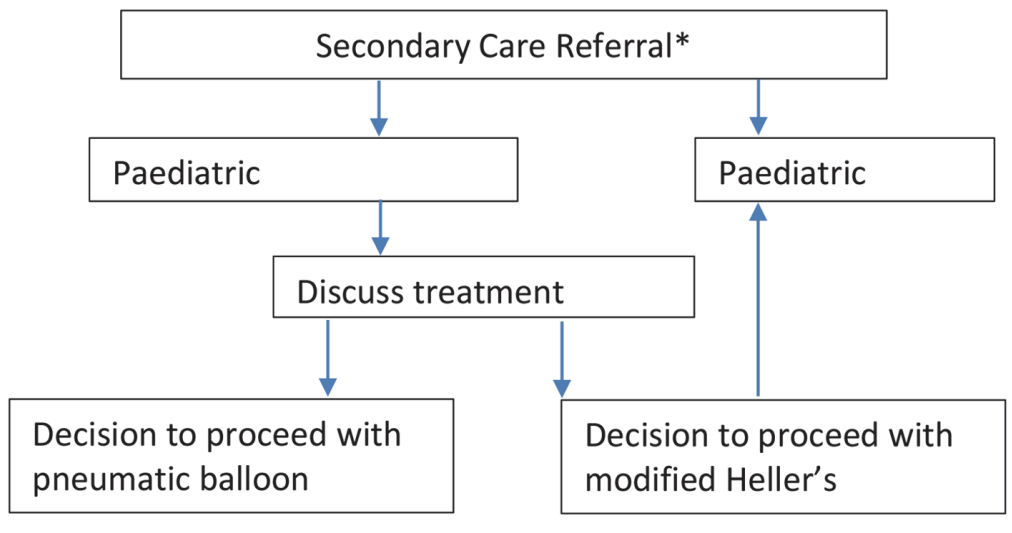

\begin{tabular}{|l|}
\hline Required \\
Investigations \\
Barium swallow- \\
to be done by \\
referring team \\
High Resolution \\
Manometry- to be \\
done in Tertiary \\
Centre
\end{tabular}

Serial pneumatic balloon dilatations (endoscopic guidance only): Eckhardt score pre-procedure

First PD- 30mm, 15Psi of air 45-60secs

Second PD (4weeks) - 35mm of air 45-60secs

Timed Barium Swallow - 1min, $2 \mathrm{~min}$ and $5 \mathrm{~min}$

Third PD $35 \mathrm{~mm} / 40 \mathrm{~mm}$ if there is evidence of delayed barium passage (even if

dysphagia score is low)

Figure 1: Current Algorithm for the Management of Paediatric Achalasia

Abstract 011 Figure 1 Current algorithm for the management of paediatric Achalaosia

${ }^{*}$ Secondary care has the option to refer initially to paediatric gastroenterology or paediatric surgery

less invasive and a day-case procedure. Other advantages include no surgical scars and ability for repeat procedures if symptoms recur. For the health service, PD is a more costeffective action compared to HM.

Methods We analysed retrospective data from all paediatric patients who underwent pneumatic balloon dilatations from 2010 to 2020 in our centre.

Results In a ten-year period, 37 pneumatic dilatations were performed on 13 patients ( 7 male, 6 female), all with endoscopic guidance only. The median age at first dilatation was 12.7 years (range 5.4-15.7). Two patients had a prior diagnosis of eosinophilic oesophagitis. One patient had been managed as having an eating disorder before finally being diagnosed with achalasia. Two patients had a previous Heller's myotomy and had been referred to our service following symptom recurrence.

Thirteen patients had diagnostic barium swallow prior to treatment, with 10/13 showing abnormalities (results for the remaining $3 / 13$ were unavailable). Oesophageal manometry (changed from standard manometry [SM]to High resolution manometry [HRM] in 2016) was performed in $7 / 13$ patients.

Four patients were classified with type 1 achalasia, 2 patients with type 2 achalasia (HRM)and the remainder were uncategorised (SM).

All patients had improvement in dysphagia, chest pain and regurgitation. However, 3 patients had a partial response to PD, with 2 proceeding to modified Heller's myotomy and 1 patient having POEM.
Only one complication was recorded, with one patient requiring post-operative admission due to vomiting (known episodic vomiting). There were no incidences of perforation.

Figure 1 shows the current algorithm for the management of paediatric achalasia in our centre.

Conclusions Recommendations for the optimal management of Achalasia have changed with time. Centres should offer equal access to PD and HM and decisions made individually, taking into account patient preference. PD in the paediatric population is safe, even when repeated sets of dilatations are needed. Achalasia is a rare disorder, and we advocate for centres with the requisite expertise to be developed as supra-regional centres.

\section{Poster presentations}

\section{P01 A CASE OF POSSIBLE AUTOIMMUNE PANCREATITIS}

${ }^{1}$ Sally Buxton, ${ }^{1}$ Paul Bellis, ${ }^{2}$ Manu Nayar, ${ }^{1}$ Bruce McLain, ${ }^{1}$ Anirban Mukhopadhyay, ${ }^{1}$ Raj S Parmar, 'Julian Thomas. 'Great North Children's Hospital, Newcastle; ${ }^{2}$ Royal Victoria Infirmary, Newcastle

\subsection{6/flgastro-2021-bspghan.12}

Introduction Autoimmune pancreatitis is a rare paediatric condition with management driven by adult guidelines. However, case reports suggest that the paediatric disease presents 